\title{
Dual CRISPR interference and activation for targeted reactivation of $X$-linked endogenous FOXP3 in human breast cancer cells
}

Xuelian Cui ${ }^{1}$, Chao Zhang ${ }^{1}$, Zhifang X ${ }^{1}$, Shuaibin Wang ${ }^{1}$, Xin Li ${ }^{1}$, Erica Stringer-Reasor ${ }^{2,3}$, Sejong Bae ${ }^{2,3}$, Leiping Zeng ${ }^{4}$, Dehua Zhao ${ }^{4}$, Runhua Liu ${ }^{1,2^{*}}$, Lei S. Qi ${ }^{4,5,6^{*}}$ and Lizhong Wang ${ }^{1,2^{*}}$ (D)

\begin{abstract}
Background: Unlike autosomal tumor suppressors, X-linked tumor suppressors can be inactivated by a single hit due to $\mathrm{X}$-chromosome inactivation $(\mathrm{XCl}$ ). Here, we argue that targeted reactivation of the non-mutated allele from XCI offers a potential therapy for female breast cancers.

Methods: Towards this goal, we developed a dual CRISPR interference and activation (CRISPRi/a) approach for simultaneously silencing and reactivating multiple X-linked genes using two orthogonal, nuclease-deficient CRISPR/Cas9 (dCas9) proteins.

Results: Using Streptococcus pyogenes dCas9-KRAB for silencing XIST and Staphylococcus aureus dCas9-VPR for activating FOXP3, we achieved CRISPR activation of FOXP3 in various cell lines of human female breast cancers. In human breast cancer HCC202 cells, which express a synonymous heterozygous mutation in the coding region of FOXP3, simultaneous silencing of XIST from XCI led to enhanced and prolonged FOXP3 activation. Also, reactivation of endogenous FOXP3 in breast cancer cells by CRISPRi/a inhibited tumor growth in vitro and in vivo. We further optimized CRISPRa by fusing dCas9 to the demethylase TET1 and observed enhanced FOXP3 activation. Analysis of the conserved CpG-rich region of FOXP3 intron 1 confirmed that CRISPRi/a-mediated simultaneous FOXP3 activation and XIST silencing were accompanied by elevated $\mathrm{H} 4$ acetylation, including $\mathrm{H} 4 \mathrm{~K} 5 \mathrm{ac}, \mathrm{H} 4 \mathrm{~K} 8 \mathrm{ac}$, and $\mathrm{H} 4 \mathrm{~K} 16 \mathrm{ac}$, and $\mathrm{H} 3 \mathrm{~K} 4 \mathrm{me} 3$ and lower DNA methylation. This indicates that CRISPRi/a targeting to XIST and FOXP3 loci alters their transcription and their nearby epigenetic modifications.
\end{abstract}

Conclusions: The simultaneous activation and repression of the X-linked, endogenous FOXP3 and XIST from XCI offers a useful research tool and a potential therapeutic for female breast cancers.

Keywords: FOXP3, Breast cancer, CRISPR, X-linked gene, Transcript

*Correspondence: runhua@uab.edu; stanley.qi@stanford.edu; Iwang12@uab. edu

${ }^{1}$ Department of Genetics, University of Alabama at Birmingham, 720 20th Street South, Birmingham, AL 35294, USA

${ }^{4}$ Department of Bioengineering, Stanford University, 443 Via Ortega, Stanford, CA 94305, USA

Full list of author information is available at the end of the article

\section{Main text}

Autosomal tumor suppressor genes can be inactivated by a two-hit Knudson mechanism. However, X chromosome-linked tumor suppressor genes, such as FOXP3 at Xp11.23 [1], can be inactivated by a single-hit mechanism, because of X-chromosome inactivation (XCI). In female breast cancer cells, all identified gene deletions of original author(s) and the source, provide a link to the Creative Commons licence, and indicate if changes were made. The images or other third party material in this article are included in the article's Creative Commons licence, unless indicated otherwise in a credit line to the material. If material is not included in the article's Creative Commons licence and your intended use is not permitted by statutory regulation or exceeds the permitted use, you will need to obtain permission directly from the copyright holder. To view a copy of this licence, visit http://creativecommons.org/licenses/by/4.0/. The Creative Commons Public Domain Dedication waiver (http://creativeco mmons.org/publicdomain/zero/1.0/) applies to the data made available in this article, unless otherwise stated in a credit line to the data. 
FOXP3 are heterozygous, and mice with a Foxp3-heterozygous mutation develop spontaneous breast cancers, suggesting that the active allele may be the only allele affected [1]. Thus, for females with cancer, it may be possible to reactivate the non-mutated, inactivated allele for therapeutic purposes. We recently developed, for complex gene regulation, a flexible endonuclease-deficient CRISPR/Cas9 (dCas9)-based platform that independently controls the expression of various genes (repression and activation) within the same cell [2]. Thus, for therapeutic purposes, targeted reactivation of XCIendogenous tumor suppressor genes may be an effective strategy to restore their function in female cancer cells. The X-linked FOXP3 gene has dual roles in tumor cells and immune cells. As a master transcriptional regulator of regulatory $\mathrm{T}$ cells, FOXP3 limits antitumor immunity [3], whereas, in breast cancer cells, it is an epithelial cell-intrinsic tumor suppressor and is implicated in a tumor-suppressive function in the inhibition of tumor initiation and progression [1, 4-6]. Thus, using FOXP3 as an X-linked model gene, we aimed to develop, for human female breast cancer cells, a tunable and reversible, targeted reactivation of $\mathrm{X}$-linked tumor suppressor genes. In the present study, using CRISPR interference and activation (CRISPRi/a), we achieved, for human female breast cancer cells, targeted reactivation of X-linked endogenous FOXP3, at least a partial reactivation from XCI. Next, we investigated the potential epigenetic mechanism during CRISPRi/a-mediated reactivation of FOXP3.

\section{Results and discussion}

\section{Transcription regulation of XIST and FOXP 3 by CRISPRi/a} in human breast cancer cells

Since most human female breast cancer cell lines have low or no FOXP3 expression [1], accompanied by heterozygous gene deletions but rare FOXP3 mutations (Table S1), it may be possible to reactivate FOXP3 from $\mathrm{XCI}$. We determined the expression levels of $X$-inactive specific transcript (XIST) in various female breast cancer cell lines, including MDA-MB-231, MCF7, and HCC202. As shown in Fig. S1, expression of XIST was high in HCC202 cells, but low in MCF7 and MDAMB-231 cells. To achieve simultaneous transcriptional repression of XIST and reactivation of FOXP3 in the same cells, we utilized two orthogonal CRISPR/dCas9 systems [2], Streptococcus pyogenes (Sp) dCas9-Krüppelassociated box (KRAB) (SpdCas9-KRAB) for silencing XIST and Staphylococcus aureus (Sa) dCas9-tripartite VP64-p65-Rta proteins (VPR) (SadCas9-VPR) for reactivation of FOXP3 (Figs. S2A and B). We established the CRISPRi/a MDA-MB-231 cell model stably expressing SadCas9-VPR and SpdCas9-KRAB (Table S2). Then, we transiently co-transduced XIST- and FOXP3-single guide
RNAs (sgRNAs) into the CRISPRi/a MDA-MB-231 cells (Figs. S2C-E). The efficacy of transduction of sgRNAs in the cells was validated by fluorescence microscopy (Fig. 1A) and Western blots (Fig. 1B). After transduction of sgRNAs, quantitative real-time PCR (qPCR) analysis showed that levels of the FOXP3 transcript were increased 8-fold by FOXP3-sgRNA with XIST-sgRNA at day 4 and up to 8 days (Fig. 1C). However, after doxycycline (Dox) addition, levels of the XIST transcript were reduced by XIST-sgRNA, but this difference was not statistically significant (Fig. 1D). Likewise, levels of the FOXP3 transcript in MDA-MB-231 cells were not significantly different after addition of Dox (Fig. 1C), suggesting a dominant activation of the FOXP3 transcript from an active X-linked allele. Furthermore, CRISPRi/a (with FOXP3/XIST-sgRNAs) and scrambled (CRISPRi/a without FOXP3/XIST-sgRNAs) MDA-MB-231 cells were injected into the fourth mammary fat pads of 8-week-old NSG female mice followed by Dox injection weekly for 28 days. As shown in Fig. S2F-H, xenograft tumor growth of CRISPRi/a MDA-MB-231 cells was slower than that of scrambled MDA-MB-231 cells. Also, reactivation of FOXP3 in xenograft tumors by CRISPRi/a was validated by qPCR (Fig. S2I). Next, we established the CRISPRi/a MCF7 cell model and transiently co-transduced both $X I S T$ - and FOXP3-sgRNAs into these cells (Fig. S3A and $B$ and Table S2). After transduction of sgRNAs, levels of the FOXP3 transcript were increased more than 8 -fold by the FOXP3-sgRNA with XIST-sgRNA (Fig. S3C). However, levels of the FOXP3 transcript in the cells were not changed by Dox, although levels of the XIST transcript were reduced by Dox (Fig. S3D), suggesting activation of the FOXP3 transcript from an active X-linked allele.

\section{CRISPRi/a-mediated targeted reactivation of X-linked FOXP3 from XCl in human breast cancer cells}

The HCC202 cell line expresses a synonymous heterozygous mutation (p.L266L

c.798G $>$ C) in the coding region of FOXP3 (Table S1), enabling us to determine, by cDNA sequencing, if FOXP3 is reactivated from one or both alleles. Also, in HCC202 cells, the two alleles of FOXP3 showed no deletion, but there were low expression levels of FOXP3 (Table S1). Thus, we established the CRISPRi/a HCC202 cell model (Fig. S4A and Table S2). After being transiently co-transduced with both XIST- and FOXP3-sgRNAs, the sgRNAtransduced cells were sorted by flow cytometry (Fig. 1E). After sgRNA transductions, levels of the FOXP3 transcript were increased approximately 6 -fold by the FOXP3sgRNA with XIST-sgRNA (Fig. 1F). After addition of Dox, transduction of XIST sgRNA reduced more than 90\% of XIST expression at day 2 and up to day 10 (Fig. 1G and $\mathrm{H})$. Simultaneously, levels of the FOXP3 transcript 


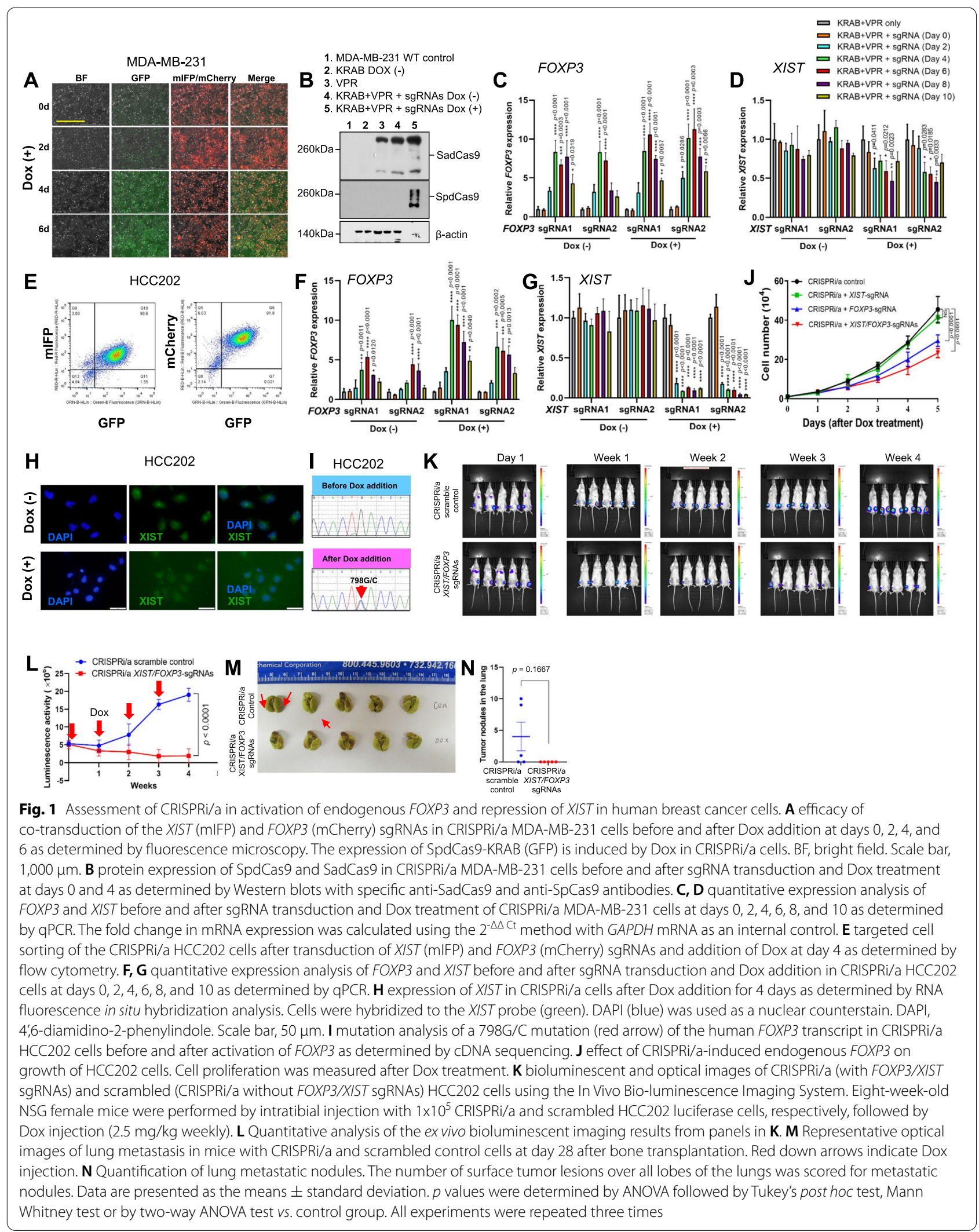


were elevated approximately 2 -fold at day 4 after Dox addition (Fig. 1F). Of note, by cDNA sequencing, a heterozygous 798G/C mutation of FOXP3 was identified in Dox-treated CRISPRi/a-FOXP3/XIST HCC202 cells but not in untreated CRISPRi/a-FOXP3/XIST HCC202 cells (Fig. 1I), suggesting that, in HCC202 cells, CRISPRi/ainduced activation of the FOXP3 transcript is at least partially reactivated from $\mathrm{XCI}$ under XIST downregulation. In addition, to test the effect of CRISPRi/a-induced FOXP3 reactivation on FOXP3 target genes in HCC202 cells, we assessed the expression of $p 21(C D K N 1 A)$ and $S K P 2$, which are prominent transcriptional targets of FOXP3 in breast cancer cells $[7,8]$. Our data revealed that, for HCC202 cells, reactivation of endogenous FOXP3 by CRISPRi/a induced the transcription of $p 21$ but reduced the transcription of SKP2 (Fig. S4B and C). Of note, Dox-induced reactivation of FOXP3 from XCI enhanced the transcriptional regulation of $p 21$ and SKP2 in $\mathrm{HCC} 202$ cells.

Using the established CRISPRi/a HCC202 cells, we determined the effect of CRISPRi/a-induced endogenous FOXP3 on cell growth. We transiently transduced XIST sgRNA, FOXP3 sgRNA, or both into CRISPRi/a HCC202 cells for 48 hours, and then added Dox to the cells for 5 days. As shown in Figs. S4D and E, levels of the FOXP3 transcript were gradually elevated in the CRISPRi/a cells with FOXP3 sgRNA, whereas levels of XIST were reduced in the CRISPRi/a cells with XIST sgRNA from days 1 to 5 after addition of Dox. Cell growth was slower for CRISPRa cells with FOXP3 sgRNA and slowest for CRISPRi/a cells with FOXP3/XIST sgRNAs relative to CRISPRi/a control cells, but there was no difference between cells with XIST sgRNA alone and control CRISPRi/a HCC202 cells (Fig. 1J), suggesting that CRISPRi/a-induced endogenous FOXP3 inhibits growth of HCC202 cells. Furthermore, we injected CRISPRi/a HCC202 cells with or without FOXP3/XIST-sgRNAs into the fourth mammary fat pads of 8-week-old NSG female mice. However, we failed to generate orthotopic xenograft tumors using CRISPRi/a and scrambled HCC202 cells until day 28 after injection. Since bone is a common metastatic site for patients with breast cancer [9], we injected the luciferase-transduced CRISPRi/a and scrambled HCC202 cells into the tibia bones of 8-week-old NSG female mice followed by weekly Dox injections. As shown in Fig. 1K and $\mathrm{L}$, luciferase imaging analysis showed that xenograft tumor growth in the bone was slower for CRISPRi/a cells (CRISPRi/a with FOXP3/XIST-sgRNAs) compared to scramble cells (CRISPRi/a without FOXP3/XIST-sgRNAs) for 28 days after tumor cell injection, supporting tumor growth inhibition by CRISPRi/a-induced endogenous FOXP3 in vivo. Likewise, lung metastases were evident in $60 \%$ of the mice (3/5) with CRISPRi/a cells but were not observed in mice (0/5) injected with scramble cells (Fig. 1M). However, due to a small sample size, quantitative analysis of tumor nodules in the lung showed no significant difference between mice with CRISPRi/a cells and scramble cells ( $p=0.1667$, Fig. $1 \mathrm{~N})$.

To exclude off-target effects of our CRISPRi/a, we evaluated the potential off-target genes of our designed sgRNAs using the Cas-OFFinder (Table S3a-d). For FOXP3-sgRNA, the three nucleotide mismatched genes, CFAP61, ERI3, and ZFAT, were assessed by qPCR. Although expression levels of the potential off-target genes undulated in the CRISPRi/a cells with FOXP3 sgRNAs, overall changes in these genes were not significant from days 0 to 10 after Dox addition (Fig. S5A-C). Likewise, for XIST-sgRNA, the three nucleotide mismatched genes, $A S X L 2$, IGF2BP2, and VAMP4, were not significantly changed from days 0 to 10 after Dox addition (Fig. S5D-F).

\section{Effect of DNA demethylation on CRISPRi/a-mediated activation of FOXP3 in human breast cancer cells}

XIST RNA works in concert with DNA methylation and histone modifications to maintain XCI $[10,11]$. In regulatory $\mathrm{T}$ cells, DNA demethylation of the conserved non-coding sequence (CNS) of the FOXP3 intron 1 is specific for inducing or stabilizing transcription of FOXP3 [12-15]. First, we determined whether treatment with the DNA methylation inhibitor, 5-aza-2'-deoxycytidine (5-Aza-CdR), enhanced CRISPRi/a-mediated activation of FOXP3 in human breast cancer cells. As shown in Fig. S6A and B, we transiently transduced FOXP3-sgRNAs into CRISPRa HCC202 cells, followed by treatment with or without 5-Aza-CdR. For the $10 \mathrm{CpG}$ sites of conserved CNS of FOXP3 intron 1, pyrosequencing analysis showed deregulation of DNA methylation by CRISPRa, 5-Aza$\mathrm{CdR}$, or both, in $7 / 10 \mathrm{CpG}$ sites, but these changes appeared to be not statistically significant (Figs. 2A and S7A-C). Likewise, FOXP3 was induced in the cells after FOXP3-sgRNA transduction, but levels of the FOXP3 transcript were not changed by treatment with 5-Aza-CdR (Fig. 2B). Next, we transiently co-transduced FOXP3/XIST-sgRNAs into CRISPRi/a HCC202 cells, followed by treatment with or without 5-Aza$\mathrm{CdR}$ and Dox addition (Fig. S6C and D). Treatment of FOXP3/XIST-sgRNAs-transduced CRISPRi/a cells with 5-Aza-CdR enhanced levels of the FOXP3 transcript approximately 2-fold at days 2 and 4 (Fig. 2C), suggesting that induction of FOXP3 by 5 -Aza-CdR from XCI was under XIST downregulation. Likewise, pyrosequencing analysis revealed deregulation of DNA methylation by CRISPRi/a, 5-Aza-CdR, or both in most CpG sites (Figs. 2A and S7D). 


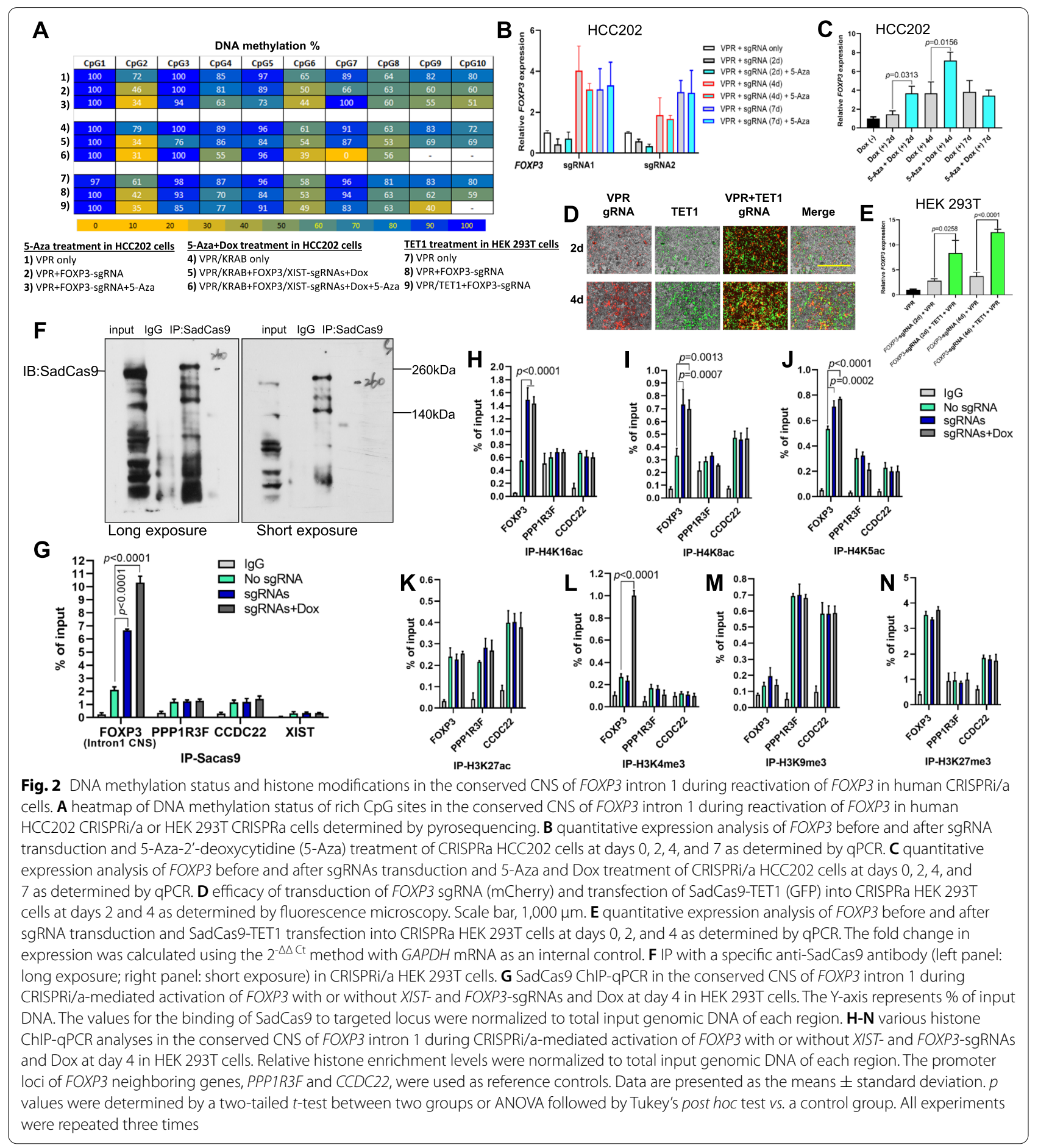

The ten-eleven translocation (TET) family of DNA demethylase proteins converts cytosine methylated at C5 $(5 \mathrm{mC})$ to $5 \mathrm{hmC}, 5 \mathrm{fC}$, and $5 \mathrm{caC}$, and finally to cytosine with the aid of thymine-DNA glycosylase [16]. These changes are associated with elevated gene transcription [17]. Thus, we constructed SadCas9-TET1 (Fig. S8A and
B) for targeted DNA demethylation in the FOXP3 CNS locus to enhance the activation of FOXP3 in human breast cancer cells. However, we failed to transfect SadCas9-TET1 into the CRISPRa HCC202 cells due to the large construct size. Next, we transfected the SadCas9TET1 and transduced the FOXP3-sgRNA into CRISPRa 
HEK 293T cells (Fig. S6E). For these cells, the efficacies of transfection and transduction were validated by fluorescence microscopy (Fig. 2D). After 2 days of transfection, Western blots confirmed protein expression of SadCas9TET1 in the transfected cells (Fig. S8C). On days 2 and 4, levels of the FOXP3 transcript were elevated approximately 3-fold in SadCas9-TET1-transfected and FOXP3sgRNA transduced cells relative to cells transduced with FOXP3-sgRNA (Fig. 2E), suggesting, for HEK 293T cells, synergistically enhanced activation of FOXP3 by coexpression of TET1 and VPR. Likewise, pyrosequencing analyses validated, for most CpG sites, deregulation of DNA methylation by CRISPRa with VPR, TET1, or both (Figs. 2A and S7E).

\section{Histone modifications during CRISPRi/a-mediated activation of FOXP3 in human breast cancer cells}

Histone methylation and acetylation either repress or activate transcription [18]. First, using established the CRISPRi/a HEK 293T cell models (Fig. S9A-D and Table S2), we performed chromatin immunoprecipitation (ChIP)-qPCR assays with a SadCas9-specific antibody (Fig. 2F). As shown in Fig. 2G, binding of SadCas9-VPR to the intron $1 \mathrm{CNS}$ locus of FOXP3 was elevated more than 3-fold in cells transduced with FOXP3/XIST-sgRNAs relative to cells without sgRNAs; this binding was enhanced after addition of Dox to the cells. Although SadCas9-VPR also bound to FOXP3 neighbor genes, $P P P 1 R 3 F$ and $C C D C 22$, these bindings were not elevated after transduction of FOXP3/XIST-sgRNAs or addition of Dox to the cells (Fig. 2G). Further, expressions of PPP1R3F and CCDC22 in the cells were not changed after the transduction of FOXP3/XIST-sgRNAs and addition of Dox (Fig. S10A and B). In addition, no specific binding of SadCas9-VPR was evident in the XIST locus (Fig. 2G). These data suggest a FOXP3-sgRNA-guided specific binding of SadCas9-VPR to the intron 1 CNS locus of FOXP3.

To address the histone modification in the intron 1 CNS locus of FOXP3 during activation of FOXP3, we performed a ChIP-qPCR assay using CRISPRa-FOXP3/ XIST-sgRNAs HEK 293T cells. As shown in Fig. 2H$\mathrm{N}$, in CRISPRi/a cells after FOXP3/XIST-sgRNAs transduction and Dox addition, H4K5ac, H4K8ac, and H4K16ac were enriched in the intron 1 CNS locus of FOXP3 but not in the promoter regions of PPP1R3F and $C C D C 22$. Of note, during activation of FOXP3, $\mathrm{H} 4 \mathrm{~K} 8 \mathrm{ac}$ and H4K16ac were elevated more than 2-fold in the intron $1 \mathrm{CNS}$ locus (Fig. $2 \mathrm{H}$ and I), but there were no significant changes after Dox addition. H3K4me3, H3K9me3, H3K27me3, and H3K27ac were minimally changed in the FOXP3, PPP1R3F, and CCDC22 loci after FOXP3/XIST-sgRNAs transduction (Fig. $2 \mathrm{~K}-\mathrm{N}$ ).
However, H3K4me3 was elevated more than 3-fold in the intron 1 CNS locus after addition of Dox (Fig. 2L). These data suggest that, during activation of FOXP3, FOXP3-sgRNA guided specific $\mathrm{H} 4$ acetylation at active alleles and $\mathrm{H} 3 \mathrm{~K} 4$ methylation at inactive alleles in the intron 1 CNS locus of FOXP3.

As reported here, we developed, for human female breast cancer cells, a CRISPRi/a approach for targeted transcriptional regulation of specific X-linked FOXP3, using two orthogonal dCas9-fusion systems, including SpdCas9-KRAB for CRISPRi to the XIST locus and SadCas9-VPR for CRISPRa to the FOXP3 locus (Fig. S11). The targeted reactivation of endogenous FOXP3 from $\mathrm{XCI}$ was achieved by simultaneous use of CRISPRi/a. Of note, targeted reactivation of FOXP3 inhibited growth of human female breast cancer cells. Furthermore, we optimized our CRISPRa system with the TET1 catalytic domain to enhance the transcriptional activation of FOXP3. The CRISPRi/a-mediated activation of FOXP3 was accompanied by $\mathrm{H} 4$ acetylation at active alleles, including H4K5ac, H4K8ac, and H4K16ac, and H3K4 methylation at inactive alleles in the intron $1 \mathrm{CNS}$ locus of FOXP3, indicating a CRISPRi/a-mediated epigenetic mechanism during activation of FOXP3.

\section{Conclusions}

The present study provides a better understanding of the CRISPRi/a-mediated activation of X-linked endogenous FOXP3 and its regulatory mechanism in human female breast cancer cells. Also, our identification of the reactivation of the X-linked FOXP3 from XCI moves beyond an incremental advance in breast cancer therapy by a targeted reactivation of $\mathrm{X}$-linked tumor suppressor genes. Since epithelial FOXP3 is inactivated in $70 \%$ of breast cancer samples [1], our results may lead to the design of preclinical studies to develop more effective treatments for female breast cancers with FOXP3 dysfunction. In addition, this concept and tools may provide new routes of targeted therapy for other X-chromosome-linked genetic disorders.

\footnotetext{
Abbreviations

5-Aza-CdR: 5-aza-2'-deoxycytidine; ChIP: Chromatin immunoprecipitation; CNS: Conserved non-coding sequence; CRISPRa: CRISPR activation; CRISPRi: CRISPR interference; dCas9: Endonuclease-deficient CRISPR/Cas9 protein; Dox: Doxycycline; H3K4me3: Tri-methylation at the 4th lysine residue of the histone $\mathrm{H} 3$ protein; $\mathrm{H} 3 \mathrm{~K} 9 \mathrm{me} 3$ : Tri-methylation at the 9th lysine residue of the histone $\mathrm{H} 3$ protein; H3K27ac: Acetylation at the 27th lysine residue of the histone $\mathrm{H} 3$ protein; $\mathrm{H} 3 \mathrm{~K} 27$ me3: Tri-methylation at the 27th lysine residue of the histone $\mathrm{H} 3$ protein; H4K5ac: Acetylation at the 5th lysine residue of the histone $\mathrm{H} 4$ protein; H4K8ac: Acetylation at the 8th lysine residue of the histone $\mathrm{H} 4$ protein; H4K16ac: Acetylation at the 16th lysine residue of the histone H4 protein; KRAB: Krüppel-associated box; qPCR: Quantitative real-time PCR; Sa: Staphylococcus aureus; sgRNA: Single guide RNA; Sp: Streptococcus pyogenes; TET: Ten-eleven translocation; VPR: Tripartite VP64-p65-Rta proteins; $\mathrm{XCl}$ : X-chromosome inactivation; XIST: $\mathrm{X}$-inactive specific transcript.
} 


\section{Supplementary Information}

The online version contains supplementary material available at https://doi. org/10.1186/s12943-021-01472-x.

Additional file 1: Figure S1. Expression of XIST in human embryonic kidney (HEK) 239T cells and breast cancer cell lines. The expression levels of XIST were assessed by GPCR. The fold change in expression was calculated using the $2^{-\triangle \Delta \mathrm{Ct}}$ method with GAPDH mRNA as an internal control. Data are presented as means \pm standard division (SD). HEK 293T, a human embryonic kidney 293 cell line with the SV40 T-antigen; MCF7, a human estrogen receptor (ER)-positive breast cancer cell line; MDAMB-231, a human triple-negative breast cancer (TNBC) cell line; HCC202, a human epidermal growth factor receptor 2 (HER2)-positive breast cancer cell line. All experiments were repeated three times. Figure S2. CRISPRi/a DNA construction, experimental procedure, and targeted reactivation of endogenous FOXP 3 in vivo in activation of endogenous FOXP3 and repression of XIST in human breast cancer MDA-MB-231 cells. A, B diagrams showing the constructs of CRISPRi/a, including S. pyogenes (Sp) dCas9-KRAB (SpdCas9-KRAB) and S. aureus (Sa) dCas9-VPR (SadCas9-VPR) used in the experiment. $\mathbf{C}$ sgRNAs $1 / 2 / 3$ targeted to the -50 to $+300 \mathrm{bp}$ upstream of the transcription start site of the XIST locus for transcription repression. D sgRNAs 1/2/3/4/5 targeted to the two $\mathrm{CpG}$ sites of the FOXP3 proximal promoter and the intron 1 regions for transcription activation. E CRISPRi/a experimental procedure for the co-transduction of XIST (mIFP)- and FOXP3 (mCherry)-sgRNAs, Dox induction, and targeted cell sorting of SpdCas9-KRAB (GFP after Dox) and SadCas9-VPR stably expressing MDA-MB-231 cells. CRISPRi, CRISPR interference; CRISPRa, CRISPR activation; sgRNA, single guide RNA; Dox, doxycycline; KRAB, transcription repressor Krüppel associated box for CRISPRi; VPR, transcription activators VP64-p65-Rta for CRISPRa. F CRISPRi/a MDAMB-231 xenograft tumor growth in NSG mice ( $n=9 /$ group). Solid black arrows indicate Dox injections. G xenograft tumors and weights at day 28 . I the expression levels of FOXP3 in xenograft tumors were assessed by qPCR. The fold change in expression was calculated using the $2^{-\Delta \Delta C t}$ method with GAPDH mRNA as an internal control. Data are presented as means $\pm S D$. $p$ values by a two-way ANOVA or a two-tailed $t$-test. Figure S3. Assessment of CRISPRi/a to activate endogenous FOXP3 and repress XIST in human breast cancer MCF7 cells. A CRISPRi/a experimental procedure for the co-transduction of XIST (mIFP)- and FOXP3 (mCherry)sgRNAs and expression of SpdCas9-KRAB (GFP) by Dox induction in CRISPRi/a MCF7 cells. B efficacy of co-transduction of the XIST- and FOXP3-sgRNAs in CRISPRi/a cells before and after Dox induction at days 4 and 8 as determined by fluorescence microscopy. Scale bar, 1,000 $\mu \mathrm{m}$. C, D quantitative expression analysis of FOXP3 and XIST before and after sgRNA transduction and Dox induction of CRISPRi/a cells at days $0,2,4,6$, 8 , and 10 as determined by qPCR. The fold change in expression was calculated using the $2^{-\triangle \triangle C t}$ method with GAPDH mRNA as an internal control. Data are presented as the means \pm SD. $p$ values by ANOVA followed by Tukey's post hoc test vs. the KRAB+VPR-only group. All experiments were repeated three times. Figure S4. CRISPRi/a DNA construction and experimental procedure and assessment of CRISPRi/a to repress XIST in activation of endogenous FOXP3 and repression of XIST in human breast cancer HCC202 cells. A CRISPRi/a experimental procedure for the co-transduction of XIST (mIFP)- and FOXP3 (mCherry)-sgRNAs and Dox induction (GFP for SpdCas9-KRAB) in CRISPRi/a HCC202 cells. B, C quantitative expression analysis of $p 21$ and SKP2 by qPCR in CRISPRi/a HCC202 cells with or without Dox at days $0,2,4,6,8$, and 10. D, E quantitative expression analysis of FOXP3 and XIST by qPCR in CRISPRi/a HCC202 cells with or without Dox. The fold change in expression was calculated using the $2^{-\triangle \triangle \mathrm{Ct}}$ method with GAPDH mRNA as an internal control. Error bars, SD. $p$ values by a two-way ANOVA or one-way ANOVA followed by Tukey's analysis. All experiments were repeated three times. Figure S5. Assessment of potential off-target genes of FOXP3 and XIST sgRNAs in CRISPRi/a HCC202 cells. Quantitative expression analysis of the potential off-target genes of FOXP3 sgRNAs (A-C) and XIST sgRNAs (D-F) before and after sgRNA transduction and Dox induction in CRISPRi/a HCC202 cells at days $0,2,4,6,8$, and 10 as determined by qPCR. The fold change in expression was calculated using the $2^{-\triangle \triangle C t}$ method with GAPDH mRNA as an internal control. Data are presented as the means \pm SD. ${ }^{*} p<$
0.05 by one-way ANOVA followed by Tukey's post hoc test. All experiments were repeated three times. Figure $\mathbf{S 6}$. Experimental procedure and efficacy of transduction in various cell models. A the CRISPRi/a experimental procedure for the transduction of FOXP3 sgRNA (mCherry) with or without 5-Aza-CdR (5-Aza) in CRISPRa HCC202 cells. B, efficacy of transduction of FOXP3 sgRNA in CRISPRa HCC202 cells before and after 5-Aza treatment at days 2, 4, and 7 as determined by fluorescence microscopy. Scale bar, $100 \mu \mathrm{m}$. C CRISPRi/a experimental procedure for the co-transduction of XIST (mIFP)- and FOXP3 (mCherry)-sgRNAs with or without 5-Aza and Dox for CRISPRa HCC202 cells. D efficacy of co-transduction of the FOXP3 sgRNA in CRISPRa HCC202 cells before and after 5-Aza and Dox treatment at days 2, 4, and 7 as determined by fluorescence microscopy. Scale bar, 1,000 $\mu \mathrm{m}$. E CRISPRa experimental procedure for the transduction of FOXP3 sgRNA (mCherry) with or without SadCas9-TET1 (GFP) into CRISPRa HEK 293T cells. Figure S7. DNA methylation status of the rich $\mathrm{CpG}$ sites in conserved CNS of FOXP3 intron 1 during CRISPRi/a-mediated activation of FOXP3 in human female cells. A diagram of the $10 \mathrm{CpG}$ sites in conserved CNS of FOXP3 intron 1 and bisulfite PCR design for DNA methylation pyrosequencing analysis. B DNA methylation analysis by pyrosequencing for CRISPRa HCC202 cells, CRISPRi/a HCC202 cells, and CRISPRa HEK 293T cells with various treatments. Pyrosequencing was performed to measure the methylation levels at $10 \mathrm{CpG}$ sites in the conserved CNS of FOXP3 intron 1 using the PyroMark Q96 ID pyrosequencer. C-E average levels of DNA methylation in CRISPRa HCC202 cells, CRISPRi/a HCC202 cells, and CRISPRa HEK 293T cells before and after various treatments. Data are presented as the means \pm SD. 5-Aza, 5-Aza-2'-deoxycytidine. All experiments were repeated three times. Figure S8. Establishment of SadCas9-TET1 DNA constructs. A schematic construction of the SadCas9-TET1 vector used in the experiment. B horizontal gel electrophoresis analysis of bands of the TET1 catalytic domain and VPR digested from SadCas9-TET1 and SadCas9-VPR vectors, respectively. Molecular sizes of the 10-kb DNA ladder are indicated on the left side. C Protein expression of TET1 after transfection into HEK 293T cells. The SadCas9-TET1 vector was transiently transfected into HEK 293T cells. The red arrow indicates the size of the SadCas9-TET1 catalytic domain. The blue arrow indicates the full size of the endogenous TET1 protein. IB, Immunoblotting. Figure S9. Targeted activation of FOXP3 and inactivation of XIST in human embryonic kidney (HEK) 293T cells. A CRISPRi experimental procedure for the transduction of XIST sgRNA (mIFP), Dox induction, and targeted cell sorting of SpdCas9-KRAB (GFP after Dox) stably expressing HEK 293T cells. B CRISPRa experimental procedure for the transduction of FOXP3 sgRNA (mCherry), Dox induction, and targeted cell soring of SpdCas9-KRAB (GFP after Dox) stably expressing HEK 293T cells. C, D quantitative expression analysis of XIST and FOXP3 by qPCR of CRISPRi and CRISPRa cells, respectively. After Dox $(1.0 \mu \mathrm{g} / \mathrm{ml})$ induction, the expression levels of XIST and FOXP3 in the transduced cells were determined at days $0,2,4,6,8$, and 10 . The fold change in expression was calculated using the $2^{-\Delta \triangle C t}$ method with GAPDH mRNA as an internal control. Data are presented as the means \pm standard deviation (SD). $p$ values by a one-way ANOVA test. CRISPRi, CRISPR interference; CRISPRa, CRISPR activation; sgRNA, single guide RNA; Dox, doxycycline; KRAB, transcription repressor Krüppel associated box for CRISPRi; VPR, transcription activators VP64-p65-Rta for CRISPRa. All experiments were repeated three times. Figure S10. Effect of CRISPRi/a-mediated activation of FOXP3 on expression of its neighboring genes in HCC202 cells. PPP1R3F and $C C D C 22$ are two FOXP3 neighboring genes at XP11.23. Quantitative expression analysis of CCDC22 (A) and PPP1R3F (B) before and after sgRNA transduction and Dox induction in the CRISPRi/a cells at days $0,2,4,6,8$, and 10 as determined by qPCR. The fold change in expression was calculated using the $2^{-\Delta \Delta \mathrm{Ct}}$ method with GAPDH mRNA as an internal control. Data are presented as the means \pm SD. $p$ values by one-way ANOVA followed by Tukey's post hoc test. All experiments were repeated three times. Figure S11. Targeted reactivation of the X-linked endogenous FOXP3 gene from $X$ chromosome inactivation (XCI) in female cells. PRC (polycomb repressive complex) 1 or 2 recruits XIST RNA and promotes epigenetic modifications that block X-linked FOXP3 gene transcription on the inactive X chromosome. DNA binding by SadCas9-VPR (VP64/p65/Rta) and SadCas9-TET to the FOXP3 intron 1 enhancer, and subsequent epigenetic modifications, in conjunction with the SpdCas9-KRAB to the XIST promoter, reactivates X-linked FOXP3 gene transcription from XCI. 


\section{Additional file 2. Materials and Methods}

Additional file 3: Table S1. Gene copy number, mutation and expression of the FOXP3 gene in human breast cancer cell lines

Additional file 4: Table S2. CRISPR/dCas9-KRAB-, VPR-, XIST-sgRNA, and FOXP3-sgRNA stably expressed cell models

Additional file 5: Table S3a. The potential off-targets of FOXP3 accessed using the Cas-OFFinder off-target searching tool. $\mathbf{b}$. The potential offtargets of XIST accessed using the Cas-OFFinder off-target searching tool. c. The potential mismatch $\leq 4$ off-target regions accessed using the Cas-OFFinder off-target searching tool. $\mathbf{d}$. The potential mismatch $\leq 4$ offtarget regions accessed using the Cas-OFFinder off-target searching tool.

Additional file 6: Table S4. CRISPR/dCas9 DNA constructs for targeted repression of XIST and activation of FOXP3

Additional file 7: Table S5. The sequences of PCR primers and CRISPR sgRNAs used in this study

Additional file 8: Table S6. Specific primary antibodies used in this study

\section{Acknowledgements}

We thank Dr. Donald Hill for editorial assistance in preparing this manuscript.

\section{Authors' contributions}

LW, RL and LSQ designed the research approach. XC, ZX, SW, XL and RL performed the experiments. XC, RL and LW analyzed data. XC, SB and LW performed statistical analyses. LZ, DZ and LSQ provided key resources. XC made a draft of the paper. XC, ESR, RL, LSQ and LW revised and edited the paper. The author(s) read and approved the final manuscript.

\section{Funding}

This work was supported by grants from the DOD (W81XWH-17-1-0017 for L. Wang and W81XWH-17-1-0018 for L.S. Qi) and the Breast Cancer Research Foundation of Alabama (L. Wang).

\section{Availability of data and materials}

Results are based, in part, upon data generated by Cas-OFFinder (http://www. rgenome.net/cas-offinder).

\section{Declarations}

\section{Ethics approval and consent to participate}

Not applicable.

\section{Consent for publication}

All authors have agreed to publish this manuscript.

\section{Competing interests}

The authors declare that they have no competing interests.

\section{Author details}

${ }^{1}$ Department of Genetics, University of Alabama at Birmingham, 720 20th Street South, Birmingham, AL 35294, USA. ²Department of O'Neal Comprehensive Cancer Center, University of Alabama at Birmingham, 720 20th Street South, Birmingham, AL 35294, USA. ${ }^{3}$ Department of Medicine, University of Alabama at Birmingham, Birmingham, AL, USA. ${ }^{4}$ Department of Bioengineering, Stanford University, 443 Via Ortega, Stanford, CA 94305, USA. 5 Department of Chemical and Systems Biology, Stanford University, 443 Via Ortega, Stanford, CA 94305, USA. ${ }^{6}$ ChEM-H Institute, Stanford University, 443 Via Ortega, Stanford, CA 94305, USA.

Received: 11 August 2021 Accepted: 26 November 2021 Published online: 07 February 2022

\section{References}

1. Zuo T, Wang L, Morrison C, Chang X, Zhang H, Li W, et al. FOXP3 is an $X$-linked breast cancer suppressor gene and an important repressor of the HER-2/ErbB2 oncogene. Cell. 2007;129:1275-86.

2. Gao Y, Xiong X, Wong S, Charles EJ, Lim WA, Qi LS. Complex transcriptional modulation with orthogonal and inducible dCas9 regulators. Nat Methods. 2016;13:1043-9.

3. Overacre-Delgoffe AE, Vignali DAA. Treg fragility: a prerequisite for effective antitumor immunity? Cancer Immunol Res. 2018;6:882-7.

4. Liu R, Liu C, Chen D, Yang WH, Liu X, Liu CG, et al. FOXP3 controls an miR146/NFkappaB negative feedback loop that inhibits apoptosis in breast cancer cells. Cancer Res. 2015;75:1703-13.

5. Zhang C, Xu Y, Hao Q, Wang S, Li H, Li J, et al. FOXP3 suppresses breast cancer metastasis through downregulation of CD44. Int J Cancer. 2015.

6. Li W, Wang L, Katoh H, Liu R, Zheng P, Liu Y. Identification of a tumor suppressor relay between the FOXP3 and the Hippo pathways in breast and prostate cancers. Cancer Res. 2011;71:2162-71.

7. Liu R, Wang L, Chen G, Katoh H, Chen C, Liu Y, et al. FOXP3 up-regulates p21 expression by site-specific inhibition of histone deacetylase 2/histone deacetylase 4 association to the locus. Cancer Res. 2009;69:2252-9.

8. Zuo T, Liu R, Zhang H, Chang X, Liu Y, Wang L, et al. FOXP3 is a novel transcriptional repressor for the breast cancer oncogene SKP2. J Clin Invest. 2007;117:3765-73.

9. Weilbaecher KN, Guise TA, McCauley LK. Cancer to bone: a fatal attraction. Nat Rev Cancer. 2011;11:411-25.

10. Pasque V, Tchieu J, Karnik R, Uyeda M, Sadhu Dimashkie A, Case D, et al. X chromosome reactivation dynamics reveal stages of reprogramming to pluripotency. Cell. 2014;159:1681-97.

11. Plath K, Mlynarczyk-Evans S, Nusinow DA, Panning B. Xist RNA and the mechanism of X chromosome inactivation. Annu Rev Genet. 2002;36:233-78.

12. Kim HP, Leonard WJ. CREB/ATF-dependent T cell receptor-induced FoxP3 gene expression: a role for DNA methylation. J Exp Med. 2007;204:1543-51.

13. Polansky JK, Kretschmer K, Freyer J, Floess S, Garbe A, Baron U, et al. DNA methylation controls Foxp3 gene expression. Eur J Immunol. 2008;38:1654-63.

14. Zheng Y, Josefowicz S, Chaudhry A, Peng XP, Forbush K, Rudensky AY. Role of conserved non-coding DNA elements in the Foxp3 gene in regulatory T-cell fate. Nature. 2010;463:808-12.

15. Ohkura N, Hamaguchi M, Morikawa H, Sugimura K, Tanaka A, Ito Y, et al. T cell receptor stimulation-induced epigenetic changes and Foxp3 expression are independent and complementary events required for Treg cell development. Immunity. 2012;37:785-99.

16. Kohli RM, Zhang Y. TET enzymes, TDG and the dynamics of DNA demethylation. Nature. 2013;502:472-9.

17. Wu H, D'Alessio AC, Ito S, Xia K, Wang Z, Cui K, et al. Dual functions of Tet1 in transcriptional regulation in mouse embryonic stem cells. Nature. 2011;473:389-93.

18. Rice JC, Briggs SD, Ueberheide B, Barber CM, Shabanowitz J, Hunt DF, et al. Histone methyltransferases direct different degrees of methylation to define distinct chromatin domains. Mol Cell. 2003;12:1591-8.

\section{Publisher's Note}

Springer Nature remains neutral with regard to jurisdictional claims in published maps and institutional affiliations. 